\title{
The Market Drivers and Business Case for Designing Accessible Public Technology
}

\author{
Dr. Lynne Coventry \\ NCR, Dundee \\ Steve Jacobs \\ Ideal Group,Inc \\ Lynne.coventry@ncr.com; steve.jacobs@ideal-group.org
}

\begin{abstract}
Public technology is often criticised for its lack of accessibility yet there is a good business case for pursing accessible design. This paper explores the market drivers for accessible design which helps to develop a business case. It also identifies some of the barriers to accessible design which are preventing companies developing accessible technology and thus realising their full market potential.
\end{abstract}

Public technology, accessibility, standards, guidelines

\section{PUBLIC TECHNOLOGY}

Public technology is a device where a consumer obtains a product or service by using a machine. In many cases the consumer must use this machine without any assistance from another person. One of the first forms of such public technology was the public telephone. Today public technology is used for many different purposes from vending consumables such as confectionary and drinks, to public information kiosks to ticket machines, cash machines and even voting terminals. In the future we will see more of these types of machines. The growth of technically controlled services changes the way of life for all people, not least for the elderly and persons with impairments. Less and less can we expect services to be delivered via a human assistant. If large groups of people are not to be excluded from access to these technologies then they must be developed with accessibility in mind. This paper will explore the business case for designing accessible public technology. It will discuss the market drivers for accessible design and the barriers which companies experience when trying to design accessible public technology.

\section{THE MARKET DRIVERS}

Historically the primary forces driving the manufacture of more accessibility products and services have been legal, moral, social and ethical. Today, manufacture of accessible products is driven by market forces. Market forces include the need to respond to consumer behaviour, the existing legislation and standards work, marketing philosophies, competition in within the market, emerging technology trends and economic expansion.

\subsection{Demographics}

The user's of technology is no longer a limited group of people. The demographics have changed by age, with an aging population of people who have grown up using technology, various levels of literacy, various languages and different experiences.

Knowing the percentage of the population, and therefore the market which has a particular disability does not necessarily help designers to design an appropriate product. If you know a user potentially has no vision, it tells you not to rely on this sense, so alternatives are required, but what capability does the person have, how do they prefer to interact with the system?

Many products and services now in common use have either started as an assistive technology, or have benefited greatly from work by the disabled community for instance on mobile phones, predictive text for sms was developed from work researchers in the area of disability, vibration mode for quiet alerts of calls when in a meeting. Speech recognition and speech synthesis originally for blind users is now being used for hands free operation of mobile phone and avatars for more social interaction, caption television for the deaf being used in bars and airports and other noisy environments.

Knowing this does not help us design more accessible products. These advances were not planned, but technology has developed to make alternatives possible. 
Through out the world there is a vast array of standards, guidelines and legislation that has a potential impact on how a particular piece of technology should be designed to be deemed to be accessible. Through out the world there are various Disability Discrimination or Human Rights legislation that is designed to prevent exclusion on the grounds of disability. The American Disability Discrimination Act has specific sections on cash machines and phone, but not on other forms of public technology. Most other countries legislation prevents discrimination, but does not assist in understanding if a particular design causes exclusion. Industry specific standards or guidelines, for instance for cash machines, prescribe the features a particular product should have to be acceptable. For instance the banking associations in different countries may adopt different standards or guidelines. There are also general purpose disability guidelines from different disability advocacy groups which suggest how different types of interfaces can be made accessible.

Fear of litigation is a driver for both the producer of the public technology and the user (i.e. the company providing the service or product via the technology). In the case of cash machines and banks, the National Federation for the Blind in the USA started to sue some banks for failing to provide access for blind users. In particular they sighted that audio lead through was possible, but the banks had chosen not to supply it. In this case the company supplying the technology could not provide audio lead through but competitors could. It should be noted that the legislation that was quoted only said that "ATMs should be independently usable by any person with a visual impairment" it did not specify how this should be achieved. This will be discussed further in barriers to accessible design.

\subsection{Desire for market share}

The biggest driver for a company these days is market share. Thus if customers are demanding accessible designs, then suppliers with more accessible designs will achieve greater market share. In the case of the US government (Section 508 of the Rehabilitation Act), they have mandated that any equipment supplied to them must be accessible, this includes technology for workers, but will also include voting terminals and public information kiosks. In the banking world there is a desire on the part of the United Nations and the World Bank to reduce poverty in developing countries. In these countries there is a growing demand to provide banking services to the "unbanked population". There is also a desire to provide other services such as Internet access via public technology as individual can not afford the technology. Many of these potential users have disabilities or are elderly.

With public technology, rather than consumer technology, the customer is not necessarily the user. The customers, if not facing litigation, may not understand the potential market they are missing by not buying accessible technology. Some technology providers also do not understand the size of the market they are potentially excluding from use of their product.

It is estimated that between 10 and 15 percent of the populations of industrial developed countries can be categorised as disabled. By 2005 it will have reached between 15 and 20 percent of the adult population. An even larger and growing proportion of the population is elderly and while not defined as disabled, may have general reduced ability associated with age. In the USA, $52 \%$ of people aged over 65 have some type of disability, while a third have a severe disability.

\subsection{Market differentiation}

As well as increasing market share by reducing exclusion, some providers may wish to differentiate themselves on the grounds of the best accessibility solution rather than just meeting basic standards.

\subsection{Indirect markets for accessible design}

Many of the solutions which are intended to improve accessibility have other advantages which also increase the size of the market for which the design is appropriate. For instance speech output for blind users may also be a more appropriate interaction method for illiterate users. While illiteracy is not a recognised disability, text based systems do exclude such users from access to the service. If a company wishes to expand into emerging markets such as India where illiteracy rates are high, having a good audio solution opens up this market. High contrast screens benefit visually impaired but also eliminates the effect of sun glare on the screen for other people. Naturalistic text to speech may be more comprehensible and it also provides additional branding opportunities.

Tourists are major users of public technology. For many English is a second language. Therefore developing content written in simplified English makes it more understandable to these users. Using simplified English ahs additional benefits, it reduces the cost of translation; it reduces ambiguity and liability of misunderstanding and it speeds reading which reduces transaction time. The use of simplified language exists in various accessibility design guidelines in support of people with cognitive and reading disabilities.

\subsection{Market Drivers Summary}


From the points made in this section, it is clear that it is possible to derive a business case for accessible public technology. The benefits are maximising the number of people who can use the product or service, access the growing population of elderly people with control of substantial wealth (monetary or knowledge), minimise the number of markets and spaces the product can not be installed, access the consumer base in emerging markets such as India, comply with accepted standards, avoid the threat of legal action and meet the going expectations and attitudes within society for more user friendly and accessibility technology. So why are companies not producing accessible technologies? The next section will look at the barriers to accessible design.

\section{BARRIERS TO ACCESSIBLE DESIGN}

\subsection{When is a product accessible?}

Many people talk about the disabled and elderly market as if it were a single market. But it is not. There are many types of disabilities, levels of disability and possible solutions to prevent exclusion. Knowing the percentage of the population, and therefore the market which has a particular disability does not necessarily help designers to design an appropriate product. If you know a user potentially has no vision, it tells you not to rely on this sense, so alternatives are required, but what capability does the person have, how do they prefer to interact with the system? So, accessibility means different things to different people. Many companies do not know what features their product should have to be acceptable. Standards and guidelines are a means for resolving this issue of diversity, by telling companies what they need to do. Standards try to help but they can be either prescriptive or vague. This gives issues for designing and evaluating designs. The requirements for accessibility for one group of users are not necessarily the same for another group. Standards can be too prescriptive, for instance the key size must be 15 $\mathrm{mm}$ by $15 \mathrm{~mm}$ and raised $3 \mathrm{~mm}$ from the surround. Does this mean that a keypad is not accessible if the key is not that size? They can also be vague, e.g. the key must be "tactilely discernable" what is tactilely discernable for a young, trained Braille user is not tactilely discernable for an elderly person with diabetes. Standards set the minimum level to achieve accessibility as agreed by committee members, they do not guarantee an optimum solution or even necessarily one which is usable. Standards arise in reaction to diversity which exists between competing products and they are too late to guide the initial technology developments.

\subsection{Incorporating accessibility requirements into product requirements}

If accessibility requirements are not part of the requirement specification for a product, they are unlikely to be designed in. Some designers, who have awareness of accessibility issues, may design in features for other purposes that assist in accessibility but they will not be able to do this if it incurs a cost and is not a specific requirement. Very few companies have accessibility representatives as part of their team, and there are different approaches to incorporating accessibility into design. Different philosophies lead to different approaches. Most companies do not have people feeding in accessibility into their requirements process.

If we were to write accessibility requirements, what form would they take? A good requirement is specific, attainable and measurable. So what would a good accessibility requirement look like?

\subsection{How to measure accessibility}

Technology is often criticised for failing to provide accessibility to certain groups. Few people ever acknowledge that accessibility is improving. As with usability, we have the problem of how to measure success. Should we test with users? Is there such a thing as a representative user? Is it tested or certified by a representative advocacy group?

\subsection{Lack of skills and awareness at appropriate levels within the companies}

Companies need to promote accessibility through all levels of the companies, from the chief executive through sales and procurement to development and testing. At many levels education is required about accessibility, the business case and the solutions. For those with responsibility for accessibility requirements and design they need to have appropriate authority to have their recommendations incorporated. There is also a lack of knowledge and skills of people to design and validate. In many companies there is maybe only a few usability or accessibility staff for an organisation with hundreds of engineers. This leaves such staff working on only a limited number of aspects of the product. With smaller organisation there may be no accessibility knowledge within the company.

Sales and buying staff need educated into what to ask for and how to promote accessibility features. Too often customers are not asking for accessibility features because they are not being sold them.

\subsection{Technology trends run counter to accessibility requirements}

Some technology trends are running counter to accessibility requirements. For instance the miniaturisation of technology we see in mobile phones can cause accessibility problems and touch screens provide no tactile feedback. The usability and accessibility of new technologies such as biometrics have not even been considered as part of the development process!

Rapid changes in technology can cause decreases in accessibility, users may be reluctant to invest in technologies with an unproven accessibility record or have proven frustrating in the past. 


\subsection{Competing requirements}

Public technology has many requirements. Many of these compete against accessibility. For instance to increase security may people want to surround the keyboard so that no one can see the keyboard. This reduces viewability and physical access for the keyboard. No one can get away from the cost requirements for technology. If accessibility components are going to increase cost, it will have to be fully justified. Cost requirements mean that the number of different versions is reduced so it would be difficult to justify different solutions for different user groups.

It is possible to have alternative approaches within a single interface; it does not have to be one size fits all anymore, but can be available in many sizes (simultaneously). However this requires understanding of the different methods and needs multiple interactions to be developed for a single product. This adds time, cost and complexity to the development process.

\section{MOVING FORWARD}

Many products and services now in common use have either started as an assistive technology, or have benefited greatly from work by the disabled community for instance on mobile phones, predictive text for sms was developed from work researchers in the area of disability, vibration mode for quiet alerts of calls when in a meeting. Speech recognition and speech synthesis originally for blind users is now being used for hands free operation of mobile phone and avatars for more social interaction, caption television for the deaf being used in bars and airports and other noisy environments. Knowing this does not help us design more accessible products. These benefits were not predicted, or planned they were accidental. However it should act as a catalyst to understand the benefits that can be derived from pursuing accessibility solutions.

The ultimate goal is to expand the market base by maximising the number of potential customer who will not be excluded from use of the product and services while not enduring excessive costs.

There is still a lot of work to be done to move forward in understanding how to do this. This includes

- Increasing the awareness of staff about accessibility issues

- Identifying the skill set required within industry to ensure accessibility is properly represented within the research and development organisations

- Ensuring accessibility is a consideration for all products and services

- Developing a standardised means of measuring accessibility of products and services

- Understand how best to include disabled people in the design process - as individuals or through user advocacy groups

- Think creatively about solving the accessibility dilemma rather than just aiming at achieving minimum compliance with standards. 